九州大学学術情報リポジトリ

Kyushu University Institutional Repository

\title{
Attractive plane curves in Differential Geometry
}

Inoguchi, Jun-ichi

Institute of Mathematics, University of Tsukuba : Professor

http://hdl. handle. net/2324/1547105

出版情報: MI lecture note series. 64, pp.121-124，2015-09-18. 九州大学マス・フォア・インダスト リ研究所

バージョン：

権利関係 : 


\title{
Attractive plane curves in Differential Geometry
}

\author{
Jun-ichi Inoguchi * \\ Institute of Mathematics \\ University of Tsukuba
}

\begin{abstract}
This is a survey talk on plane curves from differential geometric point of view and applications of plane curves to computer aided designs.
\end{abstract}

Keywords: transformation group, log-aesthetic curve, similarity geometry, similarity curvature

\section{Introduction}

Plane curves are fundamental objects in differential geometry. In industrial shape design or computer aided geometric design, curves of particular property have been used as parts of figures. In particular, plane curves with monotonous curvature function have been paid much attention. In this talk, we discuss plane curves with monotonous curvature function via similarity geometry.

\section{Euclidean geometry}

A plane curve $\boldsymbol{p}(t)=(x(t), y(t))$ is said to be regular if its derivative $\dot{\boldsymbol{p}}(t)=\mathrm{d} \boldsymbol{p} / \mathrm{d} t$ never vanishes. A regular plane curve can be reparametrised by the arc length parameter $s$. With respect to the arclength paramater, the derivative $\boldsymbol{p}^{\prime}(s):=\mathrm{d} \boldsymbol{p} / \mathrm{d} s$ is a unit vector field along the curve. Moreover the vector field $\boldsymbol{T}(s):=\boldsymbol{p}^{\prime}(s)$ and $\boldsymbol{N}(s)=R(\pi / 2) \boldsymbol{T}(s)$ constitues an orthonormal frame field $\{\boldsymbol{T}(s), \boldsymbol{N}(s)\}$ along the curve. Here $R(\theta)$ denotes the rotation matrix of rotation angle $\theta$. The orthonormal frame field is regarded as an orthogonal matrix valued function $F(s)=(\boldsymbol{T}(s) \boldsymbol{N}(s))$. The matrix valued function $F(s)$ takes value in the rotation group $\mathrm{SO}(2)$ and called the Frenet frame of the curve. The rotation group $\mathrm{SO}(2)$ is a group of all real 2 by 2 orthogonal matrices of determinant 1.

For an arclength parametrised curve $\boldsymbol{p}(s)$, its Frenet frame satisfies the Frenet equation:

$$
F^{\prime}(s)=F(s)\left(\begin{array}{cc}
0 & -\kappa(s) \\
\kappa(s) & 0
\end{array}\right) \text {. }
$$

The function $\kappa(s)$ is called the curvature of $\boldsymbol{p}(s)$.

It is well known that arclength parametrised curves are determined by the curvature function uniquely up to orientation preserving congruence transformations (Euclidean motions). Note that the set of all Euclidean motions forms a group (called the Euclidean motion group and denoted by $\mathrm{SE}(2)$ ).

\footnotetext{
*inoguchi@math.tsukuba.ac.jp
} 
In Euclidean plane geometry, there are some well known attractive curves which are characterised by the curvature function.

Example 1 (Logarithmic spirals) A logarithmic spiral is a curve parametrised as

$$
\boldsymbol{p}(t)=a\left(e^{b t} \cos t, e^{b t} \sin t\right) .
$$

Here $a$ and $b$ are positive constants. This logarithmic spiral has curvature $\kappa(s)=1 /\left(b s+a \sqrt{1+b^{2}}\right)$ with $s=a \sqrt{1+b^{2}}\left(e^{b t}-1\right) / b$. Thus logarithmic spirals are characterised as arclength parametrized curves whose curvature radius $\rho(s)=1 / \kappa(s)$ is a linear function of the arclength parameter.

Example 2 (Clothoids) The clothoid (also called the Cornu spiral) is defined as

$$
\boldsymbol{p}(s)=\left(\int_{0}^{s} \cos \frac{k s^{2}}{2} \mathrm{~d} s, \int_{0}^{s} \sin \frac{k s^{2}}{2} \mathrm{~d} s\right)
$$

by using the Fresnel integral. Here $k$ is a positive constant. clothoids have been used in highway design for many years. The clothoid has curvature $\kappa(s)=k s$.

\section{Transformation groups}

From industrial point of view, we may replace the Euclidean motion group $\mathrm{SE}(2)$ by other transformation groups strictly larger than $\mathrm{SE}(2)$ (according to the purposes or interests).

Definition 1 Let $G$ be a Lie group acting transitively on $\mathbb{R}^{2}$. The pair $\left(G, \mathbb{R}^{2}\right)$ is called a plane geometry with transformation group $G$.

The Euclidean plane geometry is formulated as $\left(\mathrm{SE}(2), \mathbb{R}^{2}\right)$.

Example 3 (Affine geometry) Let us consider the Lie group A(2) of all affine transformations on $\mathbb{R}^{2}$. Affine transformations play fundamental roles in computer graphics. The plane geometry with transformation group $\mathrm{A}(2)$ is called the affine plane geometry. As is well known, every affine transformation on $\mathbb{R}^{2}$ has the form:

$$
\boldsymbol{p} \longmapsto A \boldsymbol{p}+\boldsymbol{b},
$$

where $A$ is a real nonsingular matrix and $\boldsymbol{b}$ is a vector. See also [2].

Example 4 (Equiaffine geometry) An affine transformation: $\boldsymbol{p} \longmapsto A \boldsymbol{p}+\boldsymbol{b}$ is said to be equiaffine if $|\operatorname{det} A|=1$. The Lie group of all equiaffine transformations is called the equiaffine transformation group. The plane geometry with equiaffine transformation group is called the equiaffine plane geometry.

Take a regular curve $\boldsymbol{p}(t)$. Assume that $\boldsymbol{p}(t)$ is nondegenerate, that is, $\operatorname{det}(\dot{\boldsymbol{p}}(t) \ddot{\boldsymbol{p}}(t)) \neq 0$. Then there exists a parameter $u$ (called the equiaffine parameter) such that

$$
F^{\mathrm{SA}}(u)=\left(\frac{\mathrm{d} \boldsymbol{p}}{\mathrm{d} u}(u) \frac{\mathrm{d}^{2} \boldsymbol{p}}{\mathrm{d} u^{2}}(u)\right)
$$

has determinant 1 . Namely $F^{\mathrm{SA}}$ takes value in the special linear group $\mathrm{SL}_{2} \mathbb{R}$. Here $\mathrm{SL}_{2} \mathbb{R}$ is the Lie group of all real 2 by 2 matrices with determinant 1 . The matrix valued function $F^{\mathrm{SA}}$ is called the equiaffine frame and satisfies the equiaffine Frenet equation:

$$
\frac{\mathrm{d}}{\mathrm{d} u} F^{\mathrm{SA}}=F^{\mathrm{SA}}\left(\begin{array}{cc}
0 & -\kappa^{\mathrm{SA}}(u) \\
1 & 0
\end{array}\right) .
$$


The function $\kappa^{\mathrm{SA}}(u)$ is called the equiaffine curvature of $\boldsymbol{p}(u)$.

Analogus to Euclidean plane geometry, equiaffine parametrised curves are determined uniquely by equiaffine curvature up to orientation preserving equiaffine transformations.

Consider a parabola $\boldsymbol{p}(t)=\left(t, t^{2} / 2\right)$. Although parabolas are fundamental examples of plane curves in Euclidean plane geometry, their curvature is not simple. In fact, one can check that $\kappa(t)=$ $\left(1+t^{2}\right)^{-3 / 2}$. On the other hand in equiaffine geometry, paraboras are characterised as equiaffine curves with vanishing equiaffine curvature.

\section{Similarity geometry}

In this talk, we concentrate our attention to plane curves under similarity transformations. The plane geometry under similarity transformations is called the similarity plane geometry.

Two circles $C_{1}$ and $C_{2}$ are congruent, that is, equivalent in Euclidean plane geometry if and only if they are related by congruent transformations. Thus $C_{1}$ is congruent to $C_{2}$ if and only if the radii of two circles coincide. On the other hand in similarity plane geometry, any two circles are equivalent. Any two parabolas are mutually equivalent in similarity plane geometry.

As is well known, logarithmic spiral is a self-similar curve. It is an interesting and natural question to characterise logarithmic spiral in terms of similarity plane geometry.

In similarity geometry, any regular plane curve with non-vanishing Euclidean curvature can be reparametrised by the turning angle function $\theta=\theta(s)$. Note that $\mathrm{d} \theta / \mathrm{d} s=\kappa(s)$. Analogues to Euclidean plane geometry, $\boldsymbol{p}(\theta)$ admits a matrix valued function $F^{\mathrm{S}}(\theta)$ which takes value in the matrix group $\mathrm{CO}^{+}(2)=\{r A \mid r>0, A \in \mathrm{SO}(2)\}$. The similarity frame $F^{\mathrm{S}}(\theta)$ satisfies the similarity Frenet equation

$$
\frac{\mathrm{d}}{\mathrm{d} \theta} F^{\mathrm{S}}(\theta)=F^{\mathrm{S}}(\theta)\left(\begin{array}{cc}
-S(\theta) & -1 \\
1 & -S(\theta)
\end{array}\right) .
$$

The function $S(\theta)$ is called the Similarity curvature. One can see that $\boldsymbol{p}(\theta)$ has vanishing similarity curvature if and only if $\boldsymbol{p}(\theta)$ is a circle. Regular plane curves with non-vanishing Euclidean curvature with non-zero constant similarity curvature are equivalent to logarithmic spirals.

\section{Log aesthetic curves}

Harada [1] considered logarithmic distribution diagram of curvature (LDDC) for plane curves. They discovered that attractive plane curves drawn by car designers have approximately linear LDDC. The LDDC of an arclength parametrised curve $\boldsymbol{p}(s)$ is

$$
(X(s), Y(s))=(\log \rho(s), \log |\mathrm{d} s / \mathrm{d} \log \rho(s)|) .
$$

Miura [3] has given the following mathematical formulation of log-aesthetic curve: A log-aesthetic curve is an arclength parametrised curve $\boldsymbol{p}(s)$ whose curvature radius $\rho(s)$ is given by

$$
\rho(s)^{\alpha}=a s+b, \quad \alpha \neq 0
$$

for some constants $a$ and $b$. In case $\alpha=0$, a log-aesthetic curve is defined by the equation:

$$
\rho(s)=\exp (a s+b)
$$


for some constants $a$ and $b$. Note that the class of log-aesthetic curves contains log-spirals $(\alpha=1)$, clothoids $(\alpha=-1)$ and Nielsen spiral $(\alpha=0)$. We notice that the $Y$-coordinate of the LDDC is rewritten as $Y=X-\log |S(\theta)|$. This fact motivates us to study log-aesthetic curves in terms of similarity geometry. We have the following fundamental result.

Theorem 1 (cf. [4]) Log-aesthetic curves are characterised as plane curves whose reciprocal similarity curvature $1 / S(\theta)$ is a linear function of $\theta$.

Moreover one can see that the similarity curvature $S(\theta)$ of log-aesthetic curves satisfies the ordinary differential equation:

$$
\frac{\mathrm{d} S}{\mathrm{~d} \theta}=-(1-\alpha) S^{2} .
$$

Based on this fundamental fact, Sato and Shimizu [4] obtained similarity geometric formulation of certain generalisation of the class of log-aesthetic curves (called the $\rho$-shift generalised log-aesthetic curves). The work [4] motivates us to study attractive curves used in computer graphics or computer aided geometric design in terms of plane geometry under transformation groups strictly larger than Euclidean motion groups.

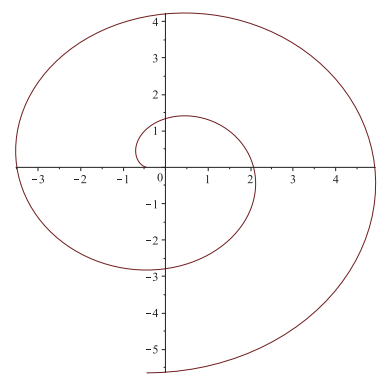

Figure 1: log-aesthetic curve with $\alpha=-1 / 4$

\section{References}

[1] T. Harada: Study of quantitative analysis of the characteristics of a curve, Forma, 12(1): 55-63, 1997.

[2] H. Ochiai and K. Anjyo: Mathematical formulation of motion and deformation and its applications, In: Mathematical Progress in Expressive Image Synthesis I, Mathematics for Industry 4, Springer Japan, 2014, pp. 123-129.

[3] K. T. Miura: A general equation of aesthetic curves and its self-affinity, Comput.-Aided Design Appl. 3: 457-464, 2006.

[4] M. Sato and Y. Shimizu: Log-aesthetic curves and Riccati equations from the viewpoint of similarity geometry, JSIAM Letters, 7: 21-24, 2014. 